\title{
On the three-dimensional configuration of electrostatic solitary waves
}

\author{
V. L. Krasovsky ${ }^{1,2}$, H. Matsumoto ${ }^{1}$, and Y. Omura ${ }^{1}$ \\ ${ }^{1}$ Radio Science Center for Space and Atmosphere, Kyoto University Uji, Kyoto 611-0011, Japan \\ ${ }^{2}$ Space Research Institute, Russian Academy of Sciences, Profsoyuznaya 84/32, Moscow 117810, Russia
}

Received: 19 January 2004 - Revised: 20 April 2004 - Accepted: 17 May 2004 - Published: 2 July 2004

\begin{abstract}
The simplest models of the electrostatic solitary waves observed by the Geotail spacecraft in the magnetosphere are developed proceeding from the concept of electron phase space holes. The technique to construct the models is based on an approximate quasi-one-dimensional description of the electron dynamics and three-dimensional analysis of the electrostatic structure of the localized wave perturbations. It is shown that the Vlasov-Poisson set of equations admits a wide diversity of model solutions of different geometry, including spatial configurations of the electrostatic potential similar to those revealed by Geotail and other spacecraft in space plasmas.
\end{abstract}

\section{Introduction}

Electrostatic solitary waves (ESW) revealed by the Geotail spacecraft in the magnetotail (Matsumoto et al., 1994) and similar electrostatic structures observed in other regions of the magnetosphere (Ergun et al., 1998; Franz et al., 1998; Bale et al., 1998; Ergun et al., 2001; Pickett et al., 2003) are often interpreted as localized Bernstein-Greene-Kruskal (BGK) modes (Bernstein et al., 1957) of the type of electron phase space holes (EH) (e.g. Berk et al., 1970; Schamel, 1979; Dupree, 1982; Omura et al., 1994; Krasovsky et al., 1997; Goldman et al., 1999; Krasovsky et al., 1999a; Franz et al., 2000; Oppenheim et al., 2001). Although the solitary waves recorded by Geotail look like almost plane perturbations or one-dimensional solitons, the solitary potential structures observed by the FAST and POLAR satellites resemble perturbations of an ellipsoidal, sometimes almost spherical shape (Ergun et al., 1998; Franz et al., 1998). It is believed that ESW may be configurated in a similar manner but typically more oblate. Thus interpretation of the localized soliton-like structures in space plasmas calls for the de-

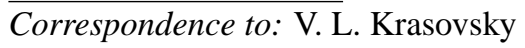

(vkrasov@mx.iki.rssi.ru) velopment of three-dimensional (3-D) models along with the approximate one-dimensional (1-D) BGK analysis.

Similar nonlinear perturbations were studied previously in magnetized laboratory plasmas (e.g. Saeki et al., 1979; Lynov et al., 1979; Guio et al., 2003) wherein electron dynamics is almost one-dimensional in nature since the external magnetic field "freezes" the transverse degrees of freedom of the electron fluid motion. The quasi-one-dimensional features of the particle dynamics justify the 1-D description of the electron holes (e.g. Schamel, 1979; Turikov, 1984) as well as the 1-D theories of the electrostatic solitons of other types in a magnetized collisionless plasma.

Three-dimensional analysis of the nonlinear localized perturbations is significantly more difficult. At the present time, only few approximate 3-D models of charge density waves are known (e.g. Zakharov and Kuznetsov, 1974; Krivoruchko et al., 1975). Three-dimensional electron holes in magnetized plasmas were considered in the papers (Schamel, 1979; Shah and Turikov, 1984) on the basis of a 1-D description of the electron dynamics. Such an approach corresponds to the leading order gyrokinetic approximation and applies to a plasma imbedded in a sufficiently strong external magnetic field. Boundary conditions being used in the 3-D EH models are characteristic of a laboratory plasma bounded by cylindrical surface of a waveguide (Lynov et al., 1979). A similar approach has been demonstrated to describe EHs in an unbounded space plasma (Chen and Parks, 2002). However, the physical meaning of the boundary condition used in this work has not been clarified. Probably, the transverse dependence of the electrostatic potential utilized in the paper (Chen and Parks, 2002) coincides with the radial dependence typical of a bounded plasma (Schamel, 1979) on a purely formal basis, and the model solutions describe the behavior of the physical quantities only in a space region confined in the transverse direction.

In this paper, we consider possible algorithms to construct the 3-D models of electron holes, assuming the electron motion to be quasi-one-dimensional similarly to the mentioned work (Schamel, 1979; Shah and Turikov, 1984; Chen and 
Parks, 2002). However, dealing with an unbounded plasma we do not introduce any additional boundary conditions except for monotonic decrease of electrostatic potential at infinity. Thus the spatial dependence of the potential is assumed to be of the form of a 3-D hump similar to the electrostatic structures observed by spacecraft.

A technique for constructing the geometrically simplest, spherical solutions of nonlinear Poisson equation is described in Sect. 2. In Sect. 3 we show that for trapped electron distribution of a particular form the Poisson equation may be reduced to linear Helmholtz equations in two regions of space. Then, to construct solutions of diversified geometric shapes, one can apply the well-known mathematical methods of the theory of partial differential equations and the mathematical theory of potential. The discussion of the models developed in the article is presented in Sect. 4.

\section{Localized electrostatic perturbations of a spherical shape}

Similarly to BGK analysis of plane waves, theoretical description of 3-D nonlinear charge density pertubations requires solution of Vlasov-Poisson set of equations. However, charged particle motion, even in static 3-D fields, is so complicated that a search of exact analytical solutions of the Vlasov equation is practically impossible (Krasovsky et al., 2004). Therefore, below we restrict ourselves to solutions of the Vlasov equation corresponding to the leading order of the gyrokinetic approximation as it has been done in the previous papers (Schamel, 1979; Shah and Turikov, 1984; Chen and Parks, 2002). Within the framework of such a simplified approach the kinetic equation for the electron distribution function integrated over transverse velocities takes a form of 1D Vlasov equation wherein the transverse coordinate plays a passive role of a parameter (e.g. Schamel, 1979). Therefore, solution of the stationary Vlasov equation and calculation of electron density entering the Poisson equation follow the well-known BGK technique (Bernstein et al., 1957).

Thus, assuming the electron fluid dynamics to be quasione-dimensional we consider 3-D models of localized timeindependent electrostatic structures of the EH type. For brevity, we introduce the following units of measurement for the physical variables,

$[r]=\lambda_{D e}, \quad\left[v_{e}\right]=V_{T e}$,

$V_{T e}=\left(T_{e} / m_{e}\right)^{1 / 2}=\omega_{p e} \lambda_{D e}$,

$\left[n_{e}\right]=n_{0}, \quad[\phi]=m_{e} V_{T e}^{2} / e, \quad[f]=n_{0} / V_{T e}$,

where $n_{0}$ is the undisturbed plasma density, $T_{e}$ is the electron temperature, $\omega_{p e}$ is the electron plasma frequency, $\lambda_{D e}$ is the Debye length, and $f$ is the electron distribution function. To avoid unnecessary unwieldiness, we restrict ourselves to pure electron perturbations, describing the ions as an immobile background. Then the electrostatic structure of the electron hole is described by the dimensionless Poisson equation

$\nabla^{2} \phi=n(\phi)=2 \int_{-\phi}^{\infty} \frac{d W f(W)}{\sqrt{2(W+\phi)}}-1$,

where $n$ is the plasma density perturbation and $W=\left(v_{z}-u\right)^{2} / 2-\phi$ is the longitudinal electron energy in the frame of reference moving with the velocity $u$ of the positive potential pulse $\phi>0$ along the $z$ axis coinciding with the direction of external magnetic field.

To emphasize the nature of the electrostatic perturbation as an electron hole, the right side of Eq. (2) may be transformed to a more demonstrative form. In particular, for weak perturbations $\phi \ll 1$ the Poisson equation may be rewritten in the following form (e.g. Dupree, 1982; Krasovsky et al., 2003)

$\nabla^{2} \phi=n(\phi)=\frac{\phi}{\lambda_{L}^{2}}-n_{H}(\phi)$,

$n_{H}=2 \int_{-\phi}^{0} \frac{d W f_{H}(W)}{\sqrt{2(W+\phi)}}$,

where $\lambda_{L}=\lambda_{L}(u)$ is the typical screening scale length depending on the EH velocity $u$ and unperturbed electron distribution $f_{0}(W)$. The last term in Eq. (3) is the contribution of the electron hole. This term, $n_{H}(\phi)>0$, describes the excess positive charge $q=n_{H}(\phi)$ caused by the deficit of the electrons trapped in the electrostatic potential well on the background of the unperturbed distribution function $f_{H}=f_{0}(0)-f_{T}>0, f_{T} \equiv f(W<0)$, where $f_{0}(0)$ is the value of $f_{0}$ at $v_{z}=u,(W=0)$. Thus Eq. (3) describes the linear screening of the effective positive charge of the hole by the plasma.

A characteristic feature of the nonlinear Eqs. (2) and (3) is the absence of explicit dependence of the right hand sides on the spatial variables. As a consequence, it is not difficult to construct particular spherically symmetric solutions of the equations. Consider, for example, a positive "hump" of the potential of a spherical shape

$\phi=A \exp \left(-r^{2} / \lambda^{2}\right)$.

The left hand side of the Poisson equation is easily represented as a function of $\phi$

$\nabla^{2} \phi=\frac{1}{r^{2}} \frac{\partial}{\partial r} r^{2} \frac{\partial \phi}{\partial r}=-\frac{2 \phi}{\lambda^{2}}\left[3+2 \ln \left(\frac{\phi}{A}\right)\right]$.

To complete the solution, it will suffice to find the distribution of the trapped electrons in the hole $f_{H}$ through the known $n_{H}(\phi)$. This can be readily done by the BGK technique (Bernstein et al., 1957). Because of this, we write out only the final result

$$
\begin{gathered}
f_{H}=\frac{(2 A w)^{1 / 2}}{\pi}\left[\frac{1}{\lambda_{L}^{2}}+\frac{2}{\lambda^{2}}(1+2 \ln (4 w))\right], \\
w \equiv|W| / A .
\end{gathered}
$$


Despite a singularity of the derivative $d f_{H} / d w$ at the separatrix $w=0$, the contribution of the hole $f_{H}$ to the trapped particle distribution $f_{T}$ vanishes at the separatrix, so that the electron distribution $f(w)$ is continuous everywhere (Krasovsky et al., 1999b). Requirement of positiveness of the trapped electron distribution function, $f_{T}=f_{0}(0)-f_{H} \geq 0$, imposes a restriction on the parameters of the electron hole

$(2 A)^{1 / 2} / \pi f_{0}(0) L^{2} \leq 1$,

$L=\lambda_{L}\left[1+2\left(\lambda_{L} / \lambda\right)^{2}(1+2 \ln 4)\right]^{-1 / 2}$.

However, for weak perturbations, $A \ll 1$, this limitation is usually not rigid.

Following the above procedure, one can construct a wide diversity of the solutions along with the Gaussian profile (5) since the trapped electron distribution is arbitrary to a large degree within the framework of the BGK analysis (see e.g. Krasovsky et al., 2003). The model of the spherical EH described by Eqs. (5) and (7) demonstrates the existence of formal solutions of the 3-D Poisson equation in combination with 1-D Vlasov equation similarly to the theoretical models of spherical ionic-sound solitons in a strongly magnetized plasma (Zakharov and Kuznetsov, 1974). Spatial dependencies of the EH electrostatic potential of a more complicated geometry are also quite admissible along with the spherical models. However, the procedural simplicity of the search for particular solutions does not guarantee their mathematical elegance. In the next section we consider an algorithm to construct the 3-D EH models of diversified geometric shape for such a specific trapped electron distribution that Eq. (3) may be reduced to a linear form.

\section{3 "Linear" models of 3-D electron holes}

The trapped electron distribution function $f_{T}(W)=f_{0}(0)-f_{H}(W),(W<0)$, does not depend formally on the boundary conditions at infinity in contrast to free (passing) particles, for which the corresponding distribution function has to coincide with the unperturbed distribution $f_{p}=f_{0}(W),(W>0)$, (e.g. Krasovsky et al., 2003). The arbitrariness of the trapped electron distribution function, characteristic of the stationary formulation of the problem, may be used for a simplification of the Poisson equation (3). In particular, the distribution function $f_{H}$ may be chosen so that the density of the hole $n_{H}(\phi)$ in Eq. (3) would depend linearly on the electrostatic potential. The corresponding explicit energy dependence of the trapped particle distribution can be found with the aid of the wellknown BGK technique (Bernstein et al., 1957; Krasovsky et al., 1999b) and may be written in the form

$f_{H}=\frac{1}{\pi \lambda_{L}^{2}}\left(1+\frac{1}{k^{2}}\right) \sqrt{-2\left(W+\phi_{*}\right)}$,

at $-A \leq W \leq-\phi_{*}$, and $f_{H}=0$ at $-\phi_{*} \leq W \leq 0$, where $k$ is some constant and $\phi_{*}$ is the value of the electrostatic potential on the EH boundary (where $f_{H}=n_{H}=0$ ) coinciding with one of the closed equipotential surfaces given by $\phi(r, \theta)=\phi_{*}$ in spherical coordinates $(r, \theta, \varphi)$, or $\phi(\rho, z)=\phi_{*}$ in cylindrical coordinates $(\rho, z, \varphi)$. Similarly to the model solution discussed in the previous section, the positiveness of the trapped electron distribution, $f_{T} \geq 0$, entails the limitation (8), with the difference that the spatial scale $L$ should be changed for

$L=\lambda_{L}\left(1-\phi_{*} / A\right)^{-1 / 4}\left(1+k^{-2}\right)^{-1 / 2}$.

Although an artificial specification of the function $f_{H}$ lacks any physical grounds and naturally narrows down the class of solutions under consideration, the choice (10) is convenient from the viewpoint of qualitative analysis of the $\mathrm{EH}$ electrostatic structure and the subsequent mathematical treatment. Indeed, substitution of Eq. (10) in Eq. (4) gives

$n_{H}=\frac{1}{\lambda_{L}^{2}}\left(1+\frac{1}{k^{2}}\right)\left(\phi-\phi_{*}\right)$,

so that Poisson's Eq. (3) turns out to be linear inside of EH

$\nabla^{2} \phi=\frac{\phi_{*}}{\lambda_{L}^{2}}-\frac{\phi-\phi_{*}}{\lambda^{2}}, \quad \phi_{*} \leq \phi \leq A, \quad \lambda \equiv k \lambda_{L}$,

as well as in the outer region

$\nabla^{2} \phi=\frac{\phi}{\lambda_{L}^{2}}, \quad 0 \leq \phi \leq \phi_{*}$,

where the Laplacian for the axially symmetric spatial distributions $\partial / \partial \varphi=0$ in spherical coordinates is given by

$\nabla^{2}=\frac{1}{r^{2}} \frac{\partial}{\partial r} r^{2} \frac{\partial}{\partial r}+\frac{1}{r^{2} \sin \theta} \frac{\partial}{\partial \theta} \sin \theta \frac{\partial}{\partial \theta}$.

The linearity of Eqs. (13) and (14) allows one to take advantage of the mathematical techniques well-known from the theory of partial differential equations and the mathematical theory of potential.

The apex of the potential "hump" is described by Eq. (13). The corresponding solutions even in $z=r \cos \theta$ can be written in the form of the series

$\phi=\phi_{*}\left(1+k^{2}\right)+\sum_{m=0}^{\infty} c_{m} j_{2 m}(r / \lambda) P_{2 m}(\cos \theta)$,

containing products of the Legendre polynomials and the spherical Bessel functions of the first kind,

$$
\begin{aligned}
& P_{0}(x)=1, \quad P_{2}=\left(3 x^{2}-1\right) / 2 \quad \ldots \quad, \\
& j_{0}(x)=\sin x / x, \\
& j_{2}=(\sin x / x)\left(3 / x^{2}-1\right)-\left(3 / x^{2}\right) \cos x, \ldots .
\end{aligned}
$$

Depending on the coefficients $c_{m}$, formula (16) describes a wide diversity of spatial configurations of the electrostatic potential. In particular, if $c_{m}=(-1)^{m}(4 m+1) c_{0}$, we arrive at the plane geometry $\phi=\phi_{*}\left(1+k^{2}\right)+c_{0} \cos (z / \lambda),(z=r \cos \theta)$. Such solutions describing the electrostatic potential of 1-D electron holes have already been considered in the previous work (Krasovsky et al., 1999b) and we will not dwell on 


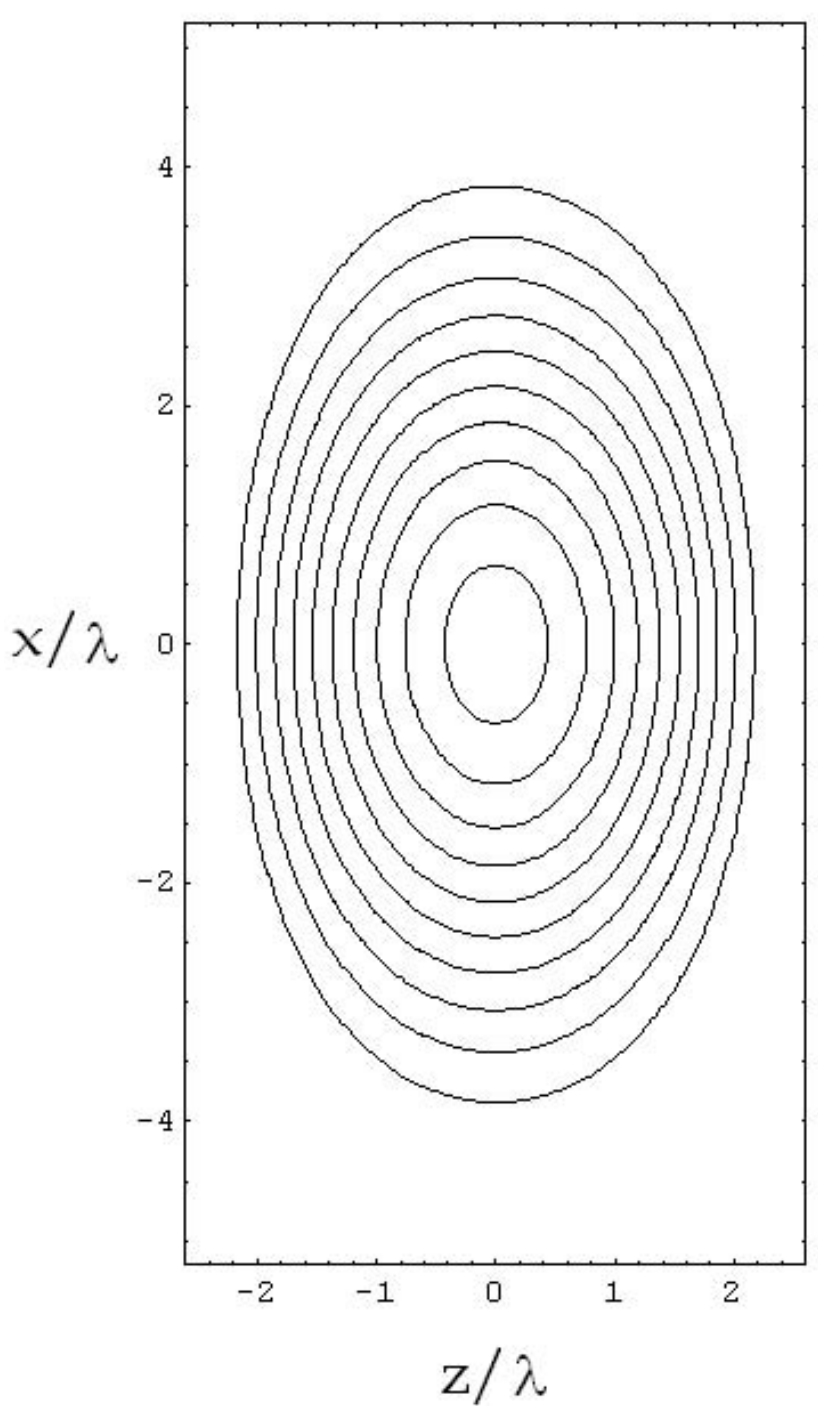

Fig. 1. Equipotential curves determined by Eq. (13) in the plane $y=0$. The corresponding equipotential surfaces in threedimensional space are given by the rotation of the plane of the paper about the axis of symmetry $z$.

them. At $c_{0} \neq 0, \quad c_{m}=0, \quad(m>0)$ expression (16) yields a spherically symmetric solution

$\phi=\phi_{*}\left(1+k^{2}\right)+c_{0}\left(\frac{\lambda}{r}\right) \sin \left(\frac{r}{\lambda}\right)$

where $c_{0}=A-\phi_{*}\left(1+k^{2}\right)$, as $\phi=A$ at $r=0$.

The solution of Eq. (14) in the exterior of the hole can be expressed in terms of special functions as well. The simplest solution is spherically symmetric solution $(\partial / \partial \theta=0)$ given by

$\phi=\phi_{*}\left(\frac{r_{*}}{r}\right) \exp \left(-\frac{r-r_{*}}{\lambda_{L}}\right)$,

where $r_{*}$ is the radius of the spherical boundary of the hole. Matching the electrostatic potential (17) and (18) and the electric field $E=-\partial \phi / \partial r$ at the boundary $r=r_{*}$ determines the interrelations between the parameters $k, \beta=r_{*} / \lambda$ and $\gamma=\phi_{*} / A$

$$
\begin{gathered}
1+k \beta+k^{2}\left(1-\beta \frac{\cos \beta}{\sin \beta}\right)=0, \\
\frac{1}{\gamma}=1+k^{2}\left(1-\frac{\beta}{\sin \beta}\right) .
\end{gathered}
$$

In the limit of small scale EH, $k \ll 1,\left(\lambda / \lambda_{L} \ll 1\right)$ we find $\gamma=\phi_{*} / A \simeq 1 / 2$ and $\beta=r_{*} / \lambda \simeq \pi$, i.e. $r_{*} / \lambda_{L} \ll 1$. In the opposite limiting case, $k \gg 1$, it follows from Eq. (19) that $\beta \simeq 3 \pi / 2, r_{*} / \lambda_{L}=\pi k \gg 1$ and $\gamma=\phi_{*} / A \ll 1$.

By varying the coefficients $c_{m}$ in Eq. (16) one can also construct solutions of a more complicated geometric shape. In particular, model solutions with concentric equipotential surfaces oblated in $z$ direction correspond geometrically to the electrostatic structures observed in space (e.g. Franz et al., 1998). One such example is the particular solution at $c_{1} / c_{0}=-1.5, c_{m}=0,(m \geq 2)$ illustrated in Fig. 1, where the shape of the equipotential curves is shown in Cartesian coordinates.

Matching the solutions at the EH boundary of an arbitrary geometrical shape is a more difficult problem in mathematical aspect since the spherical coordinates are not most convenient in the general case. However, there exist another way to calculate the spatial dependence of the potential outside of the hole. To find the spatial dependence of the potential in all space, and thereby to complete the solution, it is considerably easier to first determine a spatial dependence $\phi(r, \theta)$ with the aid of Eq. (16), to establish the shape of the boundary $r=r\left(\theta, \phi_{*}\right)$, and to find the EH density $n_{H}=\left(1+k^{2}\right)\left(\phi-\phi_{*}\right) / \lambda_{L}^{2}$. Then, taking advantage of the well-known result of the mathematical theory of potential, one can write the solution of Eq. (14) in the integral form

$\phi(\mathbf{r})=\int_{\mathcal{V}} \frac{d \mathbf{r}^{\prime} n_{H}\left(\mathbf{r}^{\prime}\right)}{4 \pi D} e^{-D / \lambda_{L}}, \quad D \equiv\left|\mathbf{r}-\mathbf{r}^{\prime}\right|, \quad\left(\phi<\phi_{*}\right)$

where the integration is performed over the volume $\mathcal{V}$ occupied by the hole, inside of which $n_{H}(\mathbf{r}) \geq 0$. Expression (20) guarantees automatically the continuity of the potential and the electric field at the EH boundary, where $\phi=\phi_{*}$, and determines the spatial dependence of the potential in the outer region. Obviously, at distances much greater than the typical spatial scales of EH, $r \gg r^{\prime}$, the electric field differs little from the field of an external pointlike charge equal in magnitude to the EH collective charge,

$\phi \simeq \frac{Q_{H}}{r} e^{-r / \lambda_{L}} \quad, \quad Q_{H}=\int_{\mathcal{V}} d \mathbf{r} n_{H}(\mathbf{r})$,

so that the equipotential surfaces grade into concentric spheres with distance from the boundary surface. This result is quite natural since the electron distribution function depends only on the energy within the framework of the simplified, qausi-one-dimensional, description of the electron motion and deviates from the unperturbed distribution $f_{0}$ only in a bounded region of space (inside of $\mathrm{EH}$ ). 


\section{Discussion}

Physical interpretation of ESW calls for the development of adequate theoretical models describing these localized wave perturbations. Although the solitary waves recorded by the Geotail spacecraft are often close to 1-D electron holes approximately described within the framework of the BGK soliton theory (Omura et al., 1994; Krasovsky et al., 1997, 2003), closer examination of their structure must take into account a more realistic 3-D geometry. In addition, in specific cases the ESW recorded by Geotail have large but finite transverse scales, i.e. deviate from 1-D models similarly to the localized electrostatic structures of a spheroidal shape detected by FAST and POLAR satellites (Ergun et al., 1998; Franz et al., 1998). Obviuosly, 1-D description of such perturbations becomes too crude.

Analytical consideration of 3-D solitary waves (even timeindependent) within the framework of the kinetic approach requires significantly more effort in comparison with 1-D problems. The main difficulty is presented by the solution of the kinetic equation since the charged particle dynamics in a 3-D electrostatic potential is described by "non-integrable" equations (Krasovsky et al., 2004). To simplify the problem, it is natural to use the gyrokinetic approximation based on the drift theory of the particle motion (e.g. Franz et al., 2000). To the first approximation, one may neglect slow drift motion, assuming electrons to be moving along magnetic field lines. Then the description of the 3-D EH reduces to the solution of the 1-D Vlasov equation and 3-D Poisson equation (Schamel, 1979; Shah and Turikov, 1984; Chen and Parks, 2002). In essence, such an approach ignores finite Larmor radius effects and corresponds to the strong magnetic field limit so that a practical application of such quasi-one-dimensional models is limited to the case of large scale perturbations as compared to the typical electron gyroradius. Nevertheless, despite some physical primitiveness, these models describe the admissible spatial configurations of the 3-D localized electrostatic potential governed by the Poisson equation and clarify the electrostatic structure of the 3-D perturbations in the strong magnetic field limit.

Above we have considered possible algorithms to construct the simplest quasi-one-dimensional models of $\mathrm{EH}$ without invoking the boundary conditions typical of a laboratory plasma bounded by a waveguide. According to the analysis performed, the set of the 1-D Vlasov and 3-D Poisson equations admits formally a wide diversity of timeindependent solutions from plane 1-D BGK solitons to spherically symmetric spatial structures, including the spatial configurations geometrically similar to those of the observed in space plasmas. Therefore, the quasi-one-dimensional EH models show that the electrostatic balance of the 3-D electron hole associated with a trapped electron deficit is formally possible. However, the geometric shape and typical scale sizes of the 3-D electrostatic perturbations remain to be rather arbitrary as in the case of the 3-D ionic-sound solitons studied by (Zakharov and Kuznetsov, 1974). On the one hand, the multiplicity of the solutions is caused by the arbi- trariness of the trapped electron distribution function characteristic of the 1-D BGK waves. On the other hand, the lack of uniqueness is closely connected with the absence of the plasma boundaries and the neglect of the finite gyroradius effects.

A refinement of the 3-D EH models calls for a more rigorous description of the electron dynamics with allowance for drift motions. In view of this it should be noted that the ordinary electric drift in an axially symmetric potential field $(\partial \phi / \partial \varphi=0)$ is of little importance in itself since the drift velocity has only azimuth component $v_{\varphi}=c E_{r} / B_{0}$, and the corresponding drift motion does not influence on the spatial distribution of the electron density entering the Poisson equation due to the symmetry of the problem. A perceptible effect on the spatial dependence of the charge density may be caused by higher order radial drift (e.g. Laframboise and Sonmor, 1993). A more careful analysis of the charged particle motion within the framework of the drift theory is a subject of further studies of the ESW structure.

Acknowledgements. This work was carried out during the stay of one of the authors (V. L. K.) at Radio Science Center for Space and Atmosphere, Kyoto University under a visiting professorship. He would like to thank Kyoto University for its warm hospitality.

Edited by: J. Büchner

Reviewed by: K. Sauer and another referee

\section{References}

Bale, S. D., Kellog, P. J., Larson, D. E., Lin, R. P., Goetz, K., and Lepping, R. P.: Bipolar electrostatic structures in the shock transition region: Evidence of electron phase space holes, Geophys. Res. Lett., 25, 2929, 1998.

Berk, H. L., Nielsen, C. E., and Roberts, K. V.: Phase space hydrodynamics of equivalent nonlinear systems: experimental and computational observations, Phys. Fluids, 13, 980, 1970.

Bernstein, I. B., Greene, J. M., and Kruskal, M. D.: Exact nonlinear plasma oscillations, Phys. Rev., 108, 546, 1957.

Chen, L.-J. and Parks, G. K.: BGK electron solitary waves: 1-D and 3-D, Nonlin. Proc. Geophys., 9, 111, 2002.

Dupree, T. H.: Theory of phase space density holes, Phys. Fluids, 25, 277, 1982.

Ergun, R. E., et al.: FAST satellite observations of large-amplitude solitary structures, Geophys. Res. Lett., 25, 2041, 1998.

Ergun, R. E., Carlson, C. W., McFadden, J. P., Strangeway, R. J., Goldman, M. V., and Newman, D. L.: Electron phase-space holes and the VLF saucer source region, Geophys. Res. Lett., 28, 3805, 2001.

Franz, J. R., Kintner, P. M., and Pickett, J. S.: POLAR observations of coherent electric field structures, Geophys. Res. Lett., 25, 1277, 1998.

Franz, J. R., Kintner, P. M., Seyler, C. E., Pickett, J. S., and Scudder, J. D.: On the perpendicular scale of electron phase-space holes, Geophys. Res. Lett., 27, 169, 2000.

Goldman, M. V., Oppenheim, M. M., and Newman, D. L.: Nonlinear two-stream instabilities as an explanation for auroral bipolar wave structures, Geophys. Res. Lett., 26, 1821, 1999. 
Guio, P., Borve, S., Daldorff, L. K. D., Lynov, J. P., Michelsen, P., Pécseli, H. L., Rasmussen, J. J., Saeki, K., and Trulsen, J.: Phase space vortices in collisionless plasma, Nonlin. Proc. Geophys., 10, 75, 2003.

Krasovsky, V. L., Matsumoto, H., and Omura, Y.: BernsteinGreene-Kruskal analysis of electrostatic solitary waves observed with Geotail, J. Geophys. Res., 102, 22 131, 1997.

Krasovsky, V. L., Matsumoto, H., and Omura, Y.: Interaction dynamics of electrostatic solitary waves, Nonlin. Proc. Geophys., 6, 205, 1999a.

Krasovsky, V. L., Matsumoto, H., and Omura, Y.: Interaction of small phase density holes, Phys. Scripta, 60, 438, 1999b.

Krasovsky, V. L., Matsumoto, H., and Omura, Y.: Electrostatic solitary waves as collective charges in magnetospheric plasma, Physical structure and properties of BGK solitons, J. Geophys. Res., 108, 1117, 2003.

Krasovsky, V. L., Matsumoto, H., and Omura, Y.: Effect of trapped particle deficit and structure of localized electrostatic perturbations of different dimensionality, J. Geophys. Res., 109, A4, A04217, doi:10.1029/2003JA00198, 2004.

Krivoruchko, S. M., Fainberg, Ya. B., Shapiro, V. D., and Shevchenko, V. I.: Solitary charge density waves in a magnetoactive plasma, Sov. Phys. JETP, Engl. Transl., 40, 1039, 1975.

Laframboise, J. G., and Sonmor, L. G.: Current collection by probes and electrodes in space magnetoplasmas: a review, J. Geophys. Res., 98, 337, 1993.

Lynov, J. P., Michelsen, P., Pécseli, H. L., Rasmussen, J. Juul, Saéki, K., and Turikov, V. A.: Observations of solitary structures in a magnetized, plasma loaded waveguide, Phys. Scripta, 20, 328, 1979.
Matsumoto, H., Kojima, H., Miyatake, T., Omura, Y., Okada, M., Nagano I., and Tsutsui, M.: Electrostatic Solitary Waves (ESW) in the magnetotail: BEN wave forms observed by GEOTAIL, Geophys. Res. Lett., 21, 2915, 1994.

Omura, Y., Kojima H., and Matsumoto, H.: Computer simulations of electrostatic solitary waves in the magnetotail: A nonlinear model of broadband electrostatic noise, Geophys. Res. Lett., 21, 2923, 1994.

Oppenheim, M. M., Vetoulis, G., Newman, D. L., and Goldman, M. V.: Evolution of electron phase-space holes in 3-D, Geophys. Res. Lett., 28, 1891, 2001.

Pickett, J. S., Menietti, J. D., Gurnett, D. A., Tsurutani, B., Kintner, P. M., Klatt, E., and Balogh, A.: Solitary potential structures observed by the Cluster spacecraft, Nonlin. Proc. Geophys., 10, 3, 2003.

Saeki, K., Michelsen, P., Pécseli, H. L., and Rasmussen, J. J.: Formation and coalescence of electron solitary holes, Phys. Rev. Lett., 42, 501, 1979.

Schamel, H.: Theory of electron holes, Phys. Scripta, 20, 336, 1979.

Shah, H. A., and Turikov, V. A.: Some properties of solitary BGK waves in a collisionless plasma, J. Plasma Phys., 31, part 3, 437, 1984.

Turikov, V. A.: Electron phase space holes as localized BGK solitons, Phys. Scripta, 30, 73, 1984.

Zakharov, V. E., and Kuznetsov, E. A.: Three-dimensional solitons, Sov. Phys. JETP, Engl. Transl., 39, 285, 1974. 